Published in final edited form as:

Arthritis Care Res (Hoboken). 2019 March ; 71(3): 427-434. doi:10.1002/acr.23607.

\title{
Gout, Hyperuricemia and Crystal-Associated Disease Network (G-CAN) consensus statement regarding labels and definitions for disease elements in gout
}

\author{
A full list of authors and affiliations appears at the end of the article.
}

\begin{abstract}
Objective-The language currently used to describe gout lacks standardisation. The aim of this project was to develop a consensus statement on the labels and definitions used to describe the basic disease elements of gout.
\end{abstract}

\begin{abstract}
Methods-Experts in gout $(\mathrm{n}=130)$ were invited to participate in a Delphi exercise and face-toface consensus meeting to reach consensus on the labelling and definitions for the basic disease elements of gout. Disease elements and labels in current use were derived from a content analysis of the contemporary medical literature, and the results of this analysis were used for item selection in the Delphi exercise and face-to-face consensus meeting.
\end{abstract}

\begin{abstract}
Results-There were 51 respondents to the Delphi exercise and 30 attendees at the face-to-face meeting. Consensus agreement ( $\ 80 \%$ ) was achieved for the labels of eight disease elements through the Delphi exercise; the remaining three labels reached consensus agreement through the face-to-face consensus meeting. The agreed labels were: monosodium urate crystals, urate, hyperuric(a)emia, tophus, subcutaneous tophus, gout flare, intercritical gout, chronic gouty arthritis, imaging evidence of monosodium urate crystal deposition, gouty bone erosion and podagra. The face-to-face meeting achieved consensus agreement for the definitions of all 11 elements and a recommendation that the label 'chronic gout' should not be used.
\end{abstract}

Conclusion-Consensus agreement was achieved for the labels and definitions of 11 elements representing the fundamental components of gout aetiology, pathophysiology and clinical presentation. The Gout, Hyperuricemia and Crystal-Associated Disease Network (G-CAN) recommends the use of these labels when describing the basic disease elements of gout.

\footnotetext{
Corresponding author: Prof Nicola Dalbeth, Bone and Joint Research Group, Department of Medicine, Faculty of Medical and Health Sciences, University of Auckland, 85 Park Rd, Grafton, Auckland 1023, New Zealand. Phone +64 93737999 x82568, Fax +64 93737677 x84377. n.dalbeth@auckland.ac.nz.

DR. WILLIAM TAYLOR (Orcid ID : 0000-0001-6075-8479)

DR. ANA BEATRIZ VARGAS-SANTOS (Orcid ID : 0000-0001-5307-0254)

DR. SEOYOUNG C. KIM (Orcid ID : 0000-0002-2517-3579)

DR. JASVINDER A SINGH (Orcid ID : 0000-0003-3485-0006)

DR. CARLO ALBERTO SCIRÈ (Orcid ID : 0000-0001-7451-0271)

Author contributions

ND (the guarantor) accepts full responsibility for the work and the conduct of the project, had access to the data, and controlled the decision to publish. ND, WJT and RT conceived of the project. DB, WJT and ND were responsible for devising the Delphi exercise surveys and the running of the face-to-face meeting, including the analysis of results. All authors participated in either or both of the Delphi exercise and face-to-face consensus meeting. DB and ND drafted the first version of the manuscript. All authors contributed to manuscript revisions and approved the final manuscript.
} 


\section{Keywords}

gout; urate; nomenclature; terminology

The language used to describe gout has substantial deficiencies in both accuracy and precision $[1,2,3]$. A recent computational analysis of the medical literature found that the terminology used for gout, its treatment and outcomes, was inconsistent, ambiguous and frequently misleading [1]. A widely accepted nomenclature for gout would have important implications for standardisation in research and clinical settings.

The Gout, Hyperuricemia and Crystal-Associated Disease Network (G-CAN) is an international, multidisciplinary collaboration, dedicated to advancing the crystal depositionassociated disorders. In recognition of the current deficits in gout terminology, G-CAN has supported the development of a Gout Nomenclature Project, aimed at establishing consensus on the labels and definitions used to describe this disease. The intended audience for this consensus statement includes health care professionals and non-physician scientists in both clinical and research settings. Herein, we describe the methodology and results of the GCAN Gout Nomenclature Project concerning the fundamental components of gout aetiology, pathophysiology and clinical presentation - referred to hereafter as the 'basic disease elements'.

\section{MATERIALS AND METHODS}

There were three stages to this project. A content analysis of the literature was undertaken to identify the basic disease elements and the labels currently used to denote them. The findings from this analysis provided a framework for the subsequent group consensus exercises. A Delphi exercise was utilized to achieve consensus agreement on the recommended labels for each disease element. A subsequent face-to-face consensus meeting had two aims: to reach agreement on any labels that had not achieved consensus through the Delphi exercise; and to reach consensus agreement on definitions for each of the disease elements. An overview of the project is depicted in Figure 1.

\section{Content analysis of the literature}

The purpose of this analysis of the literature was to identify the range of elements that collectively represent the basic disease elements of gout. Furthermore, it aimed to determine the number and frequency of labels used for each of these elements in the contemporary medical literature. This analysis has been described separately [2]. Briefly, all journal articles containing reference to gout or hyperuricaemia were examined within the ten highest ranked general rheumatology and five highest ranked general internal medicine journals (according to the 2015 Thomson-Reuters Journal Citation Reports) from $1^{\text {st }}$ January 2012 to the $31^{\text {st }}$ January 2017 , inclusive.

\section{Delphi exercise}

The principles of the Delphi technique have been described in detail elsewhere [4]. In essence, it entails a series of rounds over which proposed options are sequentially refined by 
expert opinion until consensus is met. The Delphi exercise in this project consisted of three rounds, each conducted as an online survey using Qualtrics ${ }^{\mathrm{TM}}$ software (Qualtrics, Provo, Utah). Experts in gout, identified by their membership of G-CAN, were invited by email to participate in the first survey $(n=130)$. Members were invited to participate in subsequent rounds if they had completed the preceding survey.

In each survey, the disease elements were represented using a variety of methods descriptive text, clinical photographs, diagnostic images and graphs - to accurately portray the element in question. Respondents were asked to select and rank their preferred labels for each element. The label options provided were derived from the content analysis of the literature and represented the most commonly identified labels. Labels were not included if they had been identified less than twice in the body of literature examined and no more than ten options were given for each element. There were two exceptions to this format. The label 'podagra' was unique in that there were essentially no other labels identified through the literature analysis that referred to involvement of a specific anatomical region. For this element, respondents were asked to vote whether 'podagra' was an acceptable label to denote 'an episode of acute inflammation of the first metatarsophalangeal joint' (with relevant images shown). As 'hyperuric(a)emia' was the only label used to describe 'an elevated circulating level of the final enzymatic product generated by xanthine oxidase in purine metabolism', respondents were asked to vote on whether this was an acceptable label for this element. The label options provided in the first Delphi survey are shown in Supplementary Table S1.

Respondents could comment on labels they felt strongly for or against. In accordance with the principles of the Delphi process, the second and third rounds included group feedback consisting of previous results and thematic summaries of comments provided. The label options in the second and third rounds were progressively refined depending on the achievement of consensus or other selection criteria. Consensus was defined as at least $80 \%$ of respondents selecting a label as their first choice. If consensus agreement had not been achieved for a label following the first round, it would be included as an option in the second-round if ranked within the top three by at least $20 \%$ of respondents. The two top ranked labels from the second round were included in the third round in cases where consensus had not yet been reached.

\section{Face-to-face consensus meeting}

The face-to-face meeting was held on June $14^{\text {th }}$, before the EULAR 2017 Congress, Madrid, Spain. Invitations to attend were extended to all G-CAN members. Participation in the Delphi process was not a pre-requisite for attendance. The meeting was conducted as a facilitated discussion on a list of specific questions. First, the group discussed and voted on labels for elements that had not achieved consensus through the Delphi process. All attendees were given the opportunity to express their opinion on the remaining label options; however, the introduction of new labels was not permitted. Voting rounds, conducted by 'show of hands', were held for each of these labels. Consensus was defined as at least $80 \%$ of participants in agreement for a proposed label. Attendees who abstained from voting were not included in calculating the percentage of those in agreement. 
After consensus had been achieved for all labels, the definitions for each element were addressed using the same facilitated discussion approach. Prior to the meeting, draft definitions were circulated to attendees to provide a basis for these discussions. Attendees could express their views on each element with the aim of constructing an accurate, yet concise, definition for each label. Key concepts raised were documented and incorporated into sequentially modified definitions that were then put to a 'show of hands' vote. This process continued until consensus agreement was achieved, defined as at least $80 \%$ agreeing with the proposed definition.

\section{G-CAN endorsement}

The results of the group consensus exercises and recommendations were submitted to the GCAN Board of Directors, with endorsement following their review.

\section{RESULTS}

\section{Content analysis of the literature}

Eleven basic disease elements of gout were identified; the text descriptions of these elements are shown in Table 1. Further details on the range and frequency of labels used to describe these elements have been described in detail elsewhere [2].

\section{Delphi exercise}

There were 51 G-CAN members who responded to the first survey (39\% of all members). The respondents included 19 members from Europe (37\%), 16 from North America (31\%), 11 from the Asia-Pacific region (22\%), and five from Latin America (10\%). Respondents were predominantly rheumatologists $(n=46,90 \%)$. Of the respondents who completed the first survey, 48 (94\%) completed all three rounds.

At the completion of the Delphi exercise, consensus had been achieved for labels to describe eight of the eleven basic disease elements: monosodium urate crystals, urate, hyperuric(a)emia, intercritical gout, tophus, subcutaneous tophus, imaging evidence of monosodium urate crystal deposition, and podagra (Table 2).

Consensus was not achieved through the Delphi process for the labelling of three elements. The element described as 'an episode of acute inflammation triggered by the presence of pathogenic crystals', narrowly failed to achieve consensus; $79 \%$ of respondents preferred the label 'gout flare'. For the element described as 'persistent inflammation induced by pathogenic crystals', preferences were divided between two labels; 'chronic gout' (69\%) and 'chronic gouty arthritis' (23\%). Consensus was also not achieved for the element described as 'the presence of structural bone damage due to gout'; $65 \%$ preferred the label 'bone erosion', while $33 \%$ favoured 'gouty erosion'.

\section{Face-to-face meeting}

The face-to-face consensus meeting was attended by $30 \mathrm{G}-\mathrm{CAN}$ members $(23 \%$ of all members). Twenty-four ( $80 \%$ ) of these attendees had also participated in the Delphi exercise. The panel included 17 members from Europe (57\%), six from the Asia-Pacific 
region (20\%), five from North America (17\%) and two from Latin America (7\%). Most attendees were rheumatologists ( $\mathrm{n}=28,93 \%)$. Some voting activities included only 29 attendees due to the late arrival of one member.

\section{Disease element labels}

The face-to-face meeting was successful in achieving consensus agreement on the labelling of the three unresolved elements following the Delphi exercise. These consensus element labels were: gout flare, chronic gouty arthritis and gouty bone erosion (Table 2).

Consensus for the label 'gout flare' was achieved without additional discussion, with 26 of 29 attendees $(90 \%)$ voting in favour. Group discussion identified that the text description 'persistent inflammation induced by pathogenic crystals' used in the Delphi exercise was ambiguous; this was thought to contribute to the failure to achieve consensus on the labelling of this element. Although gout-related chronic inflammation has multi-system effects, this disease element was intended to represent persistent inflammation in joint structures. Following this clarification, 24 of 29 (83\%) voted in favour of 'chronic gouty arthritis' as the preferred label. Furthermore, it was proposed that the label 'chronic gout' should be not be used. The use of the word 'chronic' in this context was felt to be redundant and potentially confusing when gout, by its very nature, is characterised by chronic crystal deposition. Consensus for the recommendation that "the label "chronic gout' should be avoided" was achieved with 28 out of 29 (97\%) voting in favour of this recommendation.

When discussing the label for the element described as 'the presence of structural bone damage due to gout', it was proposed that 'bone erosion' and 'gouty erosion' be combined to form 'gouty bone erosion'. The group considered that the use of 'gouty' was desirable for disease specificity, and to maintain consistency in this same approach used with other consensus labels. Inclusion of 'bone erosion' was judged to be in alignment with the accepted nomenclature of bone damage caused by other rheumatologic diseases. Consensus agreement for the label 'gouty bone erosion' was achieved with 27 of 29 (97\%) voting in favour.

\section{Disease element definitions}

Consensus agreement was achieved for the definitions of all 11 basic disease elements of gout (Table 3). Further details on voting results are included in Supplementary Table S2. Relevant issues arising from group discussions on the composition of these definitions are outlined here.

For 'hyperuric(a)emia', it was considered beyond the scope of this nomenclature project to include a specific threshold of blood urate concentration in its definition. Apart from the complexities involving urate solubility, and the multifactorial relationship between circulating urate levels and the clinical presentation of gout, it was argued that a specific concentration may inadvertently promote a potentially inappropriate treatment target. Therefore, the final accepted definition was modified to read 'an elevated blood urate concentration over the saturation threshold'. 
For 'gout flare', it was agreed that the definition should include the qualifying description 'a clinically evident episode' as it was deemed important to specify that such an episode was detectable either by history or physical examination. When considering 'intercritical gout', it was agreed to include the words 'gout' and 'crystal deposition' within the definition to reinforce the concept of gout as a chronic disease of crystal deposition, even in the absence of clinically evident acute inflammation. For the element labelled 'chronic gouty arthritis', it was agreed the definition should refer specifically to 'joint' involvement to maintain consistency with the use of 'arthritis' within the label.

The definition for 'imaging evidence of monosodium urate crystal deposition' was constructed to include the phrase 'findings that are highly suggestive of monosodium urate crystals on an imaging test' as crystal deposits are not always directly visualised, depending on the imaging modality used. The definition of 'gouty bone erosion' was worded to reflect the aforementioned changes in the element label. The definition explicitly refers to 'a cortical break in bone' and the gout-specific radiologic findings of an 'overhanging edge with sclerotic margins'. Even with these characteristic findings, aetiologic association is addressed as 'suggestive of gout' in recognition that causality cannot be proven.

The group recognised that the consensus definition for 'podagra' differs from the literal translation of this word (Greek: pod 'foot', agra 'seizure/trap'). However, it was accepted by most participants that this term has evolved to refer specifically to the first metatarsophalangeal joint rather than the entire first toe, foot or including the first interphalangeal joint.

\section{DISCUSSION}

We present the agreed labels and definitions for the basic disease elements of gout endorsed by G-CAN (Table 3). Our own research, consistent with that of others, has demonstrated considerable shortfalls in the terminology used to represent gout in the scientific literature $[1,2]$. The use of inconsistent, inaccurate and misleading terms has the potential to obscure interpretation of research and clinical communication. A clear and agreed terminology of the basic disease elements, which promotes an improved conceptual understanding of the disease and its pathogenesis, could also have a positive impact on patient care. The objective of this project was to address these deficits by developing a complete nomenclature for these basic elements, based on consensus expert opinion.

One of the important outcomes of this project is the agreement reached on the labelling of the element 'urate'. The common use of 'uric acid' for this element, often interchangeably with 'urate', is a potential source of confusion to clinicians, researchers and patients alike [2]. The label 'urate' is consistent with the biochemical characteristics of uric acid as a weak acid that is largely ionised at $\mathrm{pH} 7.4$, thereby making it the predominant form in blood [5].

This consensus statement also makes important recommendations on the nomenclature of elements that refer to the clinical features of gout. A key consideration is the commonly used classification of gout as being either 'acute' or 'chronic'. The label 'acute gout' suggests that the deleterious effects of this disease are limited only to those periods of intense 
inflammatory response triggered by the presence of monosodium urate crystals. In the medical literature, the label 'chronic gout' has been used by some authors to imply a distinct presentation of the disease; such as the presence of structural joint disease or extensive tophi $[6,7]$. These two commonly used labels misrepresent the pathophysiology of gout, an inherently chronic disease, in which crystal deposition is present regardless of the inflammatory state. The labels recommended by G-CAN in this consensus statement reflect contemporary understanding of disease pathophysiology. Adoption of this nomenclature may help overcome the common misperception of gout as an episodic disease which requires only acute management [8].

The label 'tophus' (Latin: 'stone') is inextricably linked to the clinical manifestations of monosodium urate (MSU) crystal deposition $[9,10]$. In this consensus statement, we recommend the label 'tophus' when referring generally to MSU deposition and its host tissue response. The label 'subcutaneous tophus' should be used for tophi evident on physical examination; the use of 'subcutaneous' is intended to add specificity while recognizing that tophi may in fact occur in other clinically-evident anatomical locations (e.g., intradermal). When referring to crystal deposits detectable by imaging, the label 'imaging evidence of monosodium urate crystal deposition' should be used. The label and definition for this element are applicable to any form of imaging; modality-specific nomenclature falls outside the scope of this project. For 'gouty bone erosion', this label and its definition recognize the characteristic appearance of bone damage in gout.

Finally, this consensus statement supports the use of 'podagra' to represent the element of a gout flare affecting the first metatarsophalangeal (MTP) joint. This is a commonly used term that has been associated with gout for many centuries $[9,10]$. Due to its historical significance and widespread acceptance, the use of this label has been deemed acceptable.

A limitation of this project is that it addresses only the basic disease elements of gout and not the name of the disease itself; nor does it deal with the nomenclature for disease staging, management and outcome measures. However, establishing a nomenclature of the basic elements was prioritized due to the foundation it provides for the labelling of more complex concepts; these are intended to be addressed in future work by G-CAN. The response rate to the group consensus exercises was modest, (39\% for the Delphi, $23 \%$ for the face-to-face consensus meeting) and participants were predominantly male (73\%). There was, however, international representation from experts in the field. A further potential limitation was the use of existing terminology identified by a content analysis of the medical literature, rather than devising novel terms. This decision was made to avoid adding to the already vast array of terms used to describe gout [2]. Refinement of established terminology was considered the most effective way of expediting widespread acceptance amongst clinicians and nonphysician researchers.

In summary, this G-CAN consensus statement regarding the labels and definitions for basic disease elements in gout represents an important step in promoting standardized nomenclature for the fundamental elements of this disease. Consensus terminology for the basic disease elements is a fundamental requirement for further work to address more advanced concepts, including the disease states of gout. The Gout, Hyperuricemia and 
Crystal-Associated Disease Network (G-CAN) recommends the use of these labels when describing the basic disease elements of gout in clinical settings and in the scientific literature, with the goal of improving the accuracy and consistency of the language used to describe gout.

\section{Supplementary Material}

Refer to Web version on PubMed Central for supplementary material.

\section{Authors}

David Bursill, MBBS ${ }^{1,2}$, William J Taylor, PhD, MBChB ${ }^{3,4}$, Robert Terkeltaub, MD ${ }^{5}$, Masanari Kuwabara, MD, $\mathrm{PhD}^{6,7}$, Tony R Merriman, $\mathrm{PhD}^{8}$, Rebecca Grainger, BMedSci, MBChB, $\mathrm{PhD}^{3,4}$, Carlos Pineda, MD, $\mathrm{PhD}^{9}$, Worawit Louthrenoo, $\mathrm{MD}^{10}, \mathrm{~N}$ Lawrence Edwards, MD, MPH ${ }^{11}$, Mariano Andrés, MD ${ }^{12,13}$, Ana Beatriz VargasSantos, $\mathrm{MD}^{14}$, Edward Roddy, MD ${ }^{15}$, Tristan Pascart, MD, $\mathrm{PhD}^{16}$, Ching-Tsai Lin, MD ${ }^{17}$, Fernando Perez-Ruiz, MD, PhD 18,19,20, Sara K Tedeschi, MD, MPH ${ }^{21,22}$, Seoyoung C Kim, MD, ScD, MSCE $21,22,23$, Leslie R Harrold, MD, MPH ${ }^{24,25}$, Geraldine McCarthy, MD ${ }^{26,27}$, Nitin Kumar, $\mathrm{PhD}^{28}$, Peter T Chapman, MD ${ }^{29}$, AnneKathrin Tausche, $\mathrm{MD}^{30}$, Janitzia Vazquez-Mellado, MD, $\mathrm{PhD}^{31,32}$, Marwin Gutierrez, $\mathrm{MD}^{33}$, Geraldo da Rocha Castelar-Pinheiro, MD, PhD ${ }^{14}$, Pascal Richette, MD, $\mathrm{PhD}^{34,35}$, Eliseo Pascual, MD, $\mathrm{PhD}^{12,13}$, Mark C Fisher, MD ${ }^{36}$, Ruben BurgosVargas, $\mathrm{MD}^{31,32}$, Philip C Robinson, $\mathrm{MBChB}, \mathrm{PhD}^{37,38}$, Jasvinder A Singh, MD, $\mathrm{MPH}^{39,40,41}$, Tim L Jansen, MD, $\mathrm{PhD}^{42}$, Kenneth G Saag, MD, $\mathrm{MSc}^{43}$, Ole Slot, $\mathrm{MD}^{44}$, Tillmann Uhlig, $\mathrm{PhD}^{45}$, Daniel H Solomon, MD, MPH 21,22,23, Robert T Keenan, MD, MPH, MBA ${ }^{46}$, Carlo Alberto Scire, MD, PhD ${ }^{47,48}$, Edyta BiernatKaluza, $\mathrm{PhD}^{49,50}$, Mats Dehlin, MD, PhD ${ }^{51}$, George Nuki, MBBS ${ }^{52}$, Naomi Schlesinger, $\mathrm{MD}^{53}$, Matthijs Janssen, $\mathrm{MD}, \mathrm{PhD}^{42}$, Lisa K Stamp, MBChB, PhD ${ }^{54}$, Francisca Sivera, $\mathrm{MD}, \mathrm{PhD}^{55}$, Anthony M Reginato, MD, $\mathrm{PhD}^{56}$, Lennart Jacobsson, $\mathrm{MD}, \mathrm{PhD}^{51}$, Frédéric Lioté, MD, PhD ${ }^{34,35}$, Hang-Korng $\mathrm{Ea}, \mathrm{MD}, \mathrm{PhD}^{34,35}$, Ann Rosenthal, MD ${ }^{57}$, Thomas Bardin, MD ${ }^{34,35}$, Hyon K Choi, MD, DrPH ${ }^{36}$, Michael S Hershfield, MD ${ }^{58}$, Christine Czegley, M.Sc ${ }^{59}$, Sung Jae Choi, MD, $\mathrm{PhD}^{60,61}$, and Nicola Dalbeth, MD ${ }^{1}$

\section{Affiliations}

${ }^{1}$ Department of Medicine, Faculty of Medical and Health Sciences, University of Auckland, Auckland, New Zealand ${ }^{2}$ Adelaide Medical School, University of Adelaide, South Australia, Australia ${ }^{3}$ Department of Medicine, University of Otago, Wellington, New Zealand ${ }^{4}$ Wellington Regional Rheumatology Unit, Hutt Valley District Health Board, Lower Hutt, New Zealand ${ }^{5}$ Veterans Affairs Medical Center, University of California, San Diego, California, USA ${ }^{6}$ Department of Cardiology, Toranomon Hospital, Tokyo, Japan ${ }^{7}$ Division of Renal Diseases and Hypertension, School of Medicine, University of Colorado Denver, Aurora, Colorado, USA ${ }^{8}$ Department of Biochemistry, University of Otago, Dunedin, New Zealand ${ }^{9}$ Instituto Nacional Rehabilitación Luis Guillermo Ibarra Ibarra, México City, México ${ }^{10}$ Division of Rheumatology, Department of Internal Medicine, Faculty of Medicine, Chiang Mai 
University, Chiang Mai, Thailand ${ }^{11}$ University of Florida College of Medicine, Gainesville, Florida, USA ${ }^{12}$ Sección de Reumatología, Hospital Universitario de Alicante, Alicante, Spain ${ }^{13}$ Departamento de Medicina Clínica, Universidad Miguel Hernández, Alicante, Spain ${ }^{14}$ Rheumatology Unit, Internal Medicine Department, State University of Rio de Janeiro, Rio de Janeiro, Brazil ${ }^{15}$ Research Institute for Primary Care and Health Sciences, Keele University, Keele, UK ${ }^{16}$ Lille Catholic University, Saint-Philibert Hospital, Department of Rheumatology, Lomme, France ${ }_{17}^{17}$ ivision of Allergy, Immunology and Rheumatology, Taichung Veterans General Hospital, Taiwan ${ }^{18}$ University of the Basque Country, Biscay, Spain ${ }^{19}$ Rheumatology Division, Cruces University Hospital, Baracaldo, Spain ${ }^{20}$ Biocruces Health Research Institute, Baracaldo, Spain ${ }^{21}$ Harvard Medical School, Boston, Massachusetts, USA ${ }^{22}$ Division of Rheumatology, Immunology and Allergy, Brigham and Women's Hospital, Boston, Massachusetts, USA ${ }^{23}$ Division of Pharmacoepidemiology and Pharmacoeconomics, Brigham and Women's Hospital, Boston, Massachusetts, USA ${ }^{24}$ University of Massachusetts Medical School, Worcester, Massachusetts, USA ${ }^{25}$ Corrona, LLC, Waltham, Massachusetts, USA ${ }^{26}$ Mater Misericordiae University Hospital, Dublin, Ireland ${ }^{27}$ University College, Dublin, Ireland ${ }^{28}$ Hypertension and Vascular Research, Henry Ford Hospital, Detroit, Michigan, USA ${ }^{29}$ Department of Rheumatology, Immunology and Allergy, Christchurch Hospital, Christchurch, New Zealand ${ }^{30}$ Department of Rheumatology, University Hospital 'Carl Gustav Carus' of the Technical University Dresden, Dresden, Germany ${ }^{31}$ Servicio de Reumatología, Hospital General de México, México City, México ${ }^{32}$ Faculty of Medicine, Universidad Nacional Autónoma de México, Ciudad de México, México ${ }^{33}$ Division of Musculoskeletal and Rheumatic Diseases, Instituto Nacional Rehabilitación, México City, México ${ }^{34}$ Department of Rhumatologie, Hôpital Lariboisière, Assistance Publique-Hopitaux de Paris, Paris, France ${ }^{35}$ INSERM UMR-1132 and Université Paris Diderot, Paris, France ${ }^{36}$ Division of Rheumatology, Allergy and Immunology, Department of Medicine, Massachusetts General Hospital, Harvard Medical School, Boston, Massachusetts, USA ${ }^{37}$ University of Queensland School of Medicine, Royal Brisbane and Women's Hospital, Queensland, Australia ${ }^{38}$ Department of Rheumatology, Royal Brisbane \& Women's Hospital, Queensland, Australia ${ }^{39}$ Medicine Service, Veterans Affairs Medical Center, Birmingham, Alabama, USA ${ }^{40}$ Department of Medicine at School of Medicine, University of Alabama at Birmingham, Birmingham, Alabama, USA ${ }^{41}$ Division of Epidemiology at School of Public Health, University of Alabama at Birmingham, Birmingham, Alabama, USA ${ }^{42}$ Department of Rheumatology, Viecuri Medical Centre, Venlo, The Netherlands ${ }^{43}$ Division of Clinical Immunology and Rheumatology, University of Alabama at Birmingham, Birmingham, Alabama, USA ${ }^{44}$ Copenhagen Center for Arthritis Research, Centre for Spinal Disorders and Rheumatology, Rigshospitalet Glostrup, Glostrup, Denmark ${ }^{45}$ Department of Rheumatology, Diakonhjemmet Hospital, Oslo, Norway ${ }^{46}$ Division of Rheumatology, Duke University School of Medicine, Durham, North Carolina, USA ${ }^{47}$ Rheumatology Unit, Department of Medical Sciences, University of Ferrara, Ferrara, Italy ${ }^{48}$ Epidemiology Unit, Italian Society for Rheumatology, Milan, Italy ${ }^{49} \mathrm{ORLIK}$, 
Outpatient Rheumatological Clinic, Warsaw, Poland ${ }^{50}$ ORLIK, Nutritional and Lifestyle Medicine Centre, Warsaw, Poland ${ }^{51}$ Department of Rheumatology and Inflammation Research, Institute of Medicine, Sahlgrenska Academy, University of Gothenburg, Sweden ${ }^{52}$ Institute for Genetics and Molecular Medicine, University of Edinburgh, Edinburgh, UK ${ }^{53}$ Department of Medicine, Rutgers - Robert Wood Johnson Medical School, New Jersey, USA ${ }^{54}$ Department of Medicine, University of Otago, Christchurch, New Zealand ${ }^{55}$ Rheumatology Unit, Hospital General Universitario de Elda, Alicante, Spain ${ }^{56}$ Division of Rheumatology, Warren Alpert School of Medicine at Brown University, Providence, Rhode Island, USA ${ }^{57}$ Medical College of Wisconsin and the Clement J. Zablocki Veterans Affairs Medical Center, Milwaukee, Wisconsin, USA ${ }^{58}$ Division of Rheumatology, Duke University Medical Center, Durham, North Carolina, USA ${ }^{59}$ Department of Internal Medicine 3, Friedrich-Alexander-University Erlangen-Nuremberg, Erlangen, Germany ${ }^{60}$ University of California, San Diego, California, USA ${ }^{61}$ Division of Rheumatology, Department of Internal Medicine, Korea University Ansan Hospital, Ansan, South Korea

\section{Acknowledgments}

Pamela Love, G-CAN Executive Director and Board Secretary, for assisting in the dissemination of the Delphi surveys and organisation of the face-to-face consensus meeting.

The survey design and for this paper were generated using Qualtrics software, Copyright () 2017 Qualtrics. Qualtrics and all other Qualtrics product or service names are registered trademarks or trademarks of Qualtrics, Provo, UT, USA. http://www.qualtrics.com

Disclosures

G-CAN has received sponsorship from Ironwood Pharmaceuticals, Horizon Pharmaceuticals, SOBI, Takeda Pharmaceutical Company, Teijin, Selecta and CymaBay. These companies had no input into the planning, implementation or interpretation of the results of this consensus project.

1. WJT has received an honorarium $(<\$ 10,000)$ from Pfizer (New Zealand) for participating in advisory board meetings.

2. RT reports research support from NIH grant AR060772, and the VA Research Service, and he is as a local site investigator the VA-CSP 594 STOP GOUT clinical trial. He also discloses research support from Ardea/Astra-Zeneca and Ironwood, and has served as a consultant for SOBI, Kowa, Horizon, and Relburn (all less than $\$ 10,000$ ), and Selecta (more than $\$ 10,000$ ).

3. TM has received research funding and consulting fees from Ardea Biosciences and consulting fees from Horizon Pharmaceuticals.

4. RG has received honoraria for speaking from AbbVie, Pfizer and Janssen and an unrestricted educational grant for research from AbbVie.

5. WL has received speaker and consultation fees from Astellas Pharma, and a speaker fee from American Taiwan Biopharm.

6. NLE has acted as a consultant for Horizon Pharmaceuticals, Ironwood Pharmaceuticals and Takeda Pharmaceutical Company.

7. MA has received consulting fees from AstraZeneca, Grünenthal and Horizon Pharmaceuticals.

8. TP has received fees from Ipsen Pharma and Mayoly Spindler.

9. FPR has engaged in advisories/speaker/educational activities for Amgen, Ardea Biosciences, AstraZeneca, Faes Farma, Grünenthal, Menarini, Horizon Pharmaceuticals and Pharmaceutical Laboratories ROVI; he has received investigation grants from Asociación de Reumatólogos de Cruces. 
10. SCK has received research grants to the Brigham and Women's Hospital from AstraZeneca, Roche, Pfizer, Bristol-Myers Squibb (BMS), and Merck.

11. LRH has stock options for Corrona, LLC. Related to gout, she has been a consultant to Pfizer in 2014 $(<\$ 10,000)$ and a consultant to MedIQ in $2015(<\$ 10,000)$. Her institution has received a grant from Takeda Pharmaceutical Company (2010-2012).

12. AKT has participated on speaker bureaus'/served on advisory boards for Berlin-Chemie, Menarini, Ipsen, SOBI, Novartis, and Ardea Biosciences/AstraZeneca.

13. JVM has participated and received fees for advisories and educational activities for Takeda Pharmaceutical Company.

14. MG has participated in advisory boards, scientific consultancies, and received consulting fees from Novartis, AbbVie, Union Chimique Belge (UCB), Esaote SpA, Janssen Pharmaceutica, Bristol-Myers Squibb (BMS) and Merck Sharp \& Dohme.

15. EP has received honoraries for talks or board meetings from Grünenthal, AstraZeneca and AMPEL Biosolutions LLC (Pegloticase).

16. MCF was a site Principal Investigator for a Selecta Biosciences study.

17. PCR has received fees $(<\$ 10,000)$ from Menarini (speaking), AstraZeneca (research funding and speaking).

18. JAS has received research grants from Takeda Pharmaceutical Company and Savient Pharmaceuticals and consultant fees from Savient, Takeda, Regeneron, Merz, Iroko, Bioiberica, Crealta/Horizon Pharmaceuticals and Allergan Pharmaceuticals, WebMD, United Business Media (UBM) LLC and the American College of Rheumatology. JAS serves as the principal investigator for an investigatorinitiated study funded by Horizon Pharmaceuticals through a grant to DINORA, Inc., a 501 (c)(3) entity. JAS is a member of the executive of OMERACT, an organization that develops outcome measures in rheumatology and receives arms-length funding from 36 companies; a member of the American College of Rheumatology's (ACR) Annual Meeting Planning Committee (AMPC); Chair of the ACR Meet-the-Professor, Workshop and Study Group Subcommittee; and a member of the Veterans Affairs Rheumatology Field Advisory Committee. JAS is the editor and the Director of the UAB Cochrane Musculoskeletal Group Satellite Center on Network Meta-analysis.

19. TLJ has received fees for speaker bureaus/lectures from AbbVie, Ardea/AstraZeneca, Bristol-Myers Squibb (BMS), Grünenthal, Janssen and Menarini. He has served on advisory boards for AbbVie, Ardea/AstraZeneca, Bristol-Myers Squibb (BMS), Celgene, Eli-Lilly, Grünenthal, Janssen, Menarini, Novartis, Pfizer, Roche, Sandoz and Union Chimique Belge (UCB). He has received grants for research projects from AbbVie, Ardea/AstraZeneca/Grünenthal.

20. KGS has served as a consultant and received research grant support from Ironwood Pharmaceuticals/ AstraZeneca, Horizon Pharmaceuticals and Takeda Pharmaceutical Company.

21. DHS has received grant support to his institution from Pfizer, grant support from Amgen, Eli Lilly, AstraZeneca, Bristol-Myers Squibb (BMS) and Genentech.

22. RTK has received advisory board/consultant fees for Horizon Pharmaceuticals, Ironwood Pharmaceuticals, AstraZeneca and received research funding from Swedish Orphan Biovitrum (SOBI).

23. CAS has participated on scientific advisory board for AstraZeneca $(<€ 3000)$ and participated in the EULAR congress for Grünenthal.

24. MD has received consulting fees from Grünenthal.

25. GN has served on advisory boards for Grünenthal, Menarini and Savient Pharmaceuticals and was a member of the Independent Data Monitoring Committee (IDMC) for Ardea/AstraZeneca trials of lesinurad.

26. NS has received grants from AstraZeneca and acted on advisory boards for Celgene, AstraZeneca and Horizon Pharmaceuticals. She has received consulting fees from Swedish Orphan Biovitrum (SOBI) and Bristol-Myers Squibb (BMS).

27. MJ has received a speakers fee from Grünenthal.

28. LKS has received grants from Ardea Biosciences.

29. FL has served as an adviser to Ardea, AstraZeneca, Grünenthal, Ipsen Pharma, Menarini and Novartis, received educational grants from Ardea, AstraZeneca, Grünenthal, Ipsen Pharma, MayolySpindler, Menarini, Novartis, Société Française de Rhumatologie and Swedish Orphan Biovitrum 
(SOBI) in support of the annual European Crystal Network (ECN) workshops, Paris, and acted as speaker for AstraZeneca, Ipsen Pharma, Mayoly-Spindler, Menarini and Novartis.

30. TB has received research grants from AstraZeneca, Ipsen and Menarini and has received fees for consultancy or talks from Ampel BioSolutions, Astellas, AstraZeneca, Biomex, Grünenthal, Ipsen, Menarini, Novartis, Savient and Swedish Orphan Biovitrum (SOBI).

31. HKC has served as a consultant for Ardea/AstraZeneca and Takeda Pharmaceutical Company.

32. MSH is a co-inventor of pegloticase (Krystexxa) and, along with Duke University, receives royalties on its sales.

33. ND discloses consulting fees, speaker fees or grants from Takeda Pharmaceutical Company, Menarini, Teijin, Amgen, Pfizer, Ardea Biosciences, AstraZeneca, Horizon Pharmaceuticals, and Cymabay.

The other authors disclose no conflicts of interest.

Funding

This research did not receive any specific grant from funding agencies in the public, commercial, or not-for-profit sectors.

\section{References}

1. Edwards NL, Malouf R, Perez-Ruiz F, Richette P, Southam S, DiChiara M. Computational lexical analysis of the language commonly used to describe gout. Arthritis Care Res (Hoboken). 2016; 68(6):763-8. [PubMed: 26414619]

2. Bursill D, Taylor W, Terkeltaub R, Dalbeth N. The nomenclature of the basic disease elements of gout: a content analysis of contemporary medical journals. Semin Arthritis Rheum. 2018

3. Simkin PA. Towards a coherent terminology of gout. Ann Rheum Dis. 1993; 52(9):693-694. [PubMed: 8239767]

4. Jones J, Hunter D. Qualitative Research: Consensus methods for medical and health services research. BMJ. 1995; 311(7001):376-380. [PubMed: 7640549]

5. Hyndman D, Liu S, Miner JN. Urate Handling in the Human Body. Curr Rheumatol Rep. 2016; 18(6):34. [PubMed: 27105641]

6. Qaseem A, Harris RP, Forciea MA. Management of acute and recurrent gout: a clinical practice guideline from the American College of Physicians. Ann Intern Med. 2017; 166(1):37-51. [PubMed: 27802478]

7. Seth R, Kydd AS, Buchbinder R, Bombardier C, Edwards CJ. Allopurinol for chronic gout. Cochrane Database Syst Rev. 2014; (10):CD006077. [PubMed: 25314636]

8. Spencer K, Carr A, Doherty M. Patient and provider barriers to effective management of gout in general practice: a qualitative study. Ann Rheum Dis. 2012; 71(9):1490-5. [PubMed: 22440822]

9. MacKenzie C. Gout and hyperuricemia: an historical perspective. Curr Treatm Opt Rheumatol. 2015; 1(2):119-130.

10. Nuki G, Simkin PA. A concise history of gout and hyperuricemia and their treatment. Arthritis Res Ther. 2006; 8(Suppl 1):S1. 


\section{Significance and Innovations}

- The terminology currently used for gout lacks standardisation and has notable deficiencies in its precision, accuracy and clarity.

- This international collaborative project provides a consensus statement on the labels and definitions given to the basic disease elements of gout.

- It is hoped that the adoption this standardised nomenclature will benefit communications in gout-related research and management. 


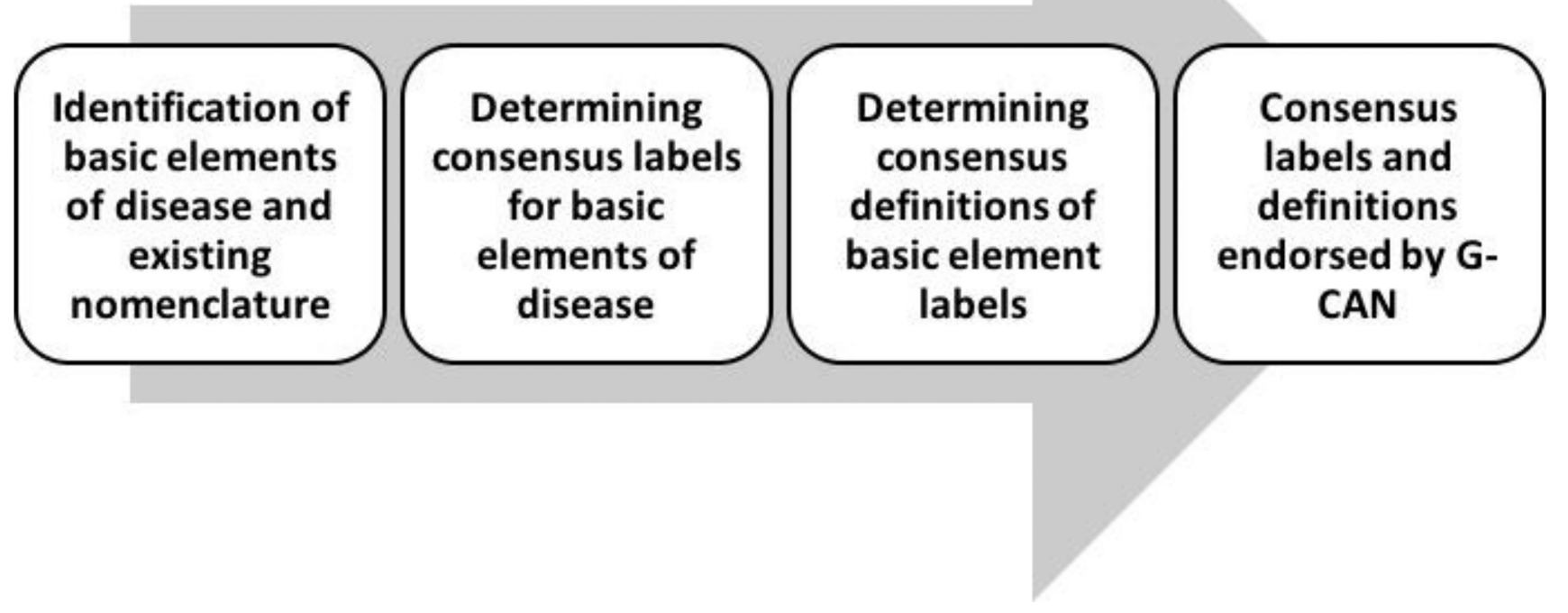

Figure 1.

Schema for the Gout Nomenclature Project. 


\section{Table 1}

Text descriptions of the basic disease elements of gout identified in a content analysis of published literature.

\begin{tabular}{l}
\hline 1. The pathogenic crystals in gout \\
2. The circulating form of the final enzymatic product generated by xanthine oxidase in purine metabolism in humans \\
\hline 3. An elevated circulating level of the final enzymatic product generated by xanthine oxidase in purine metabolism in humans \\
\hline 4. An episode of acute inflammation triggered by the presence of pathogenic crystals \\
\hline 5. The condition in which there is an absence of clinically evident inflammation after or between episodes of acute inflammation \\
\hline 6. Persistent inflammation induced by pathogenic crystals \\
\hline 7. A discrete collection of pathogenic crystals with associated host-response tissue \\
\hline 8. A discrete collection of pathogenic crystals with associated host-response tissue, detectable on physical examination \\
\hline 9. The presence of pathogenic crystal deposition on imaging \\
\hline 10. The presence of structural bone damage due to gout \\
11. An episode of acute inflammation of the 1st metatarsophalangeal joint \\
\hline
\end{tabular}


Bursill et al.

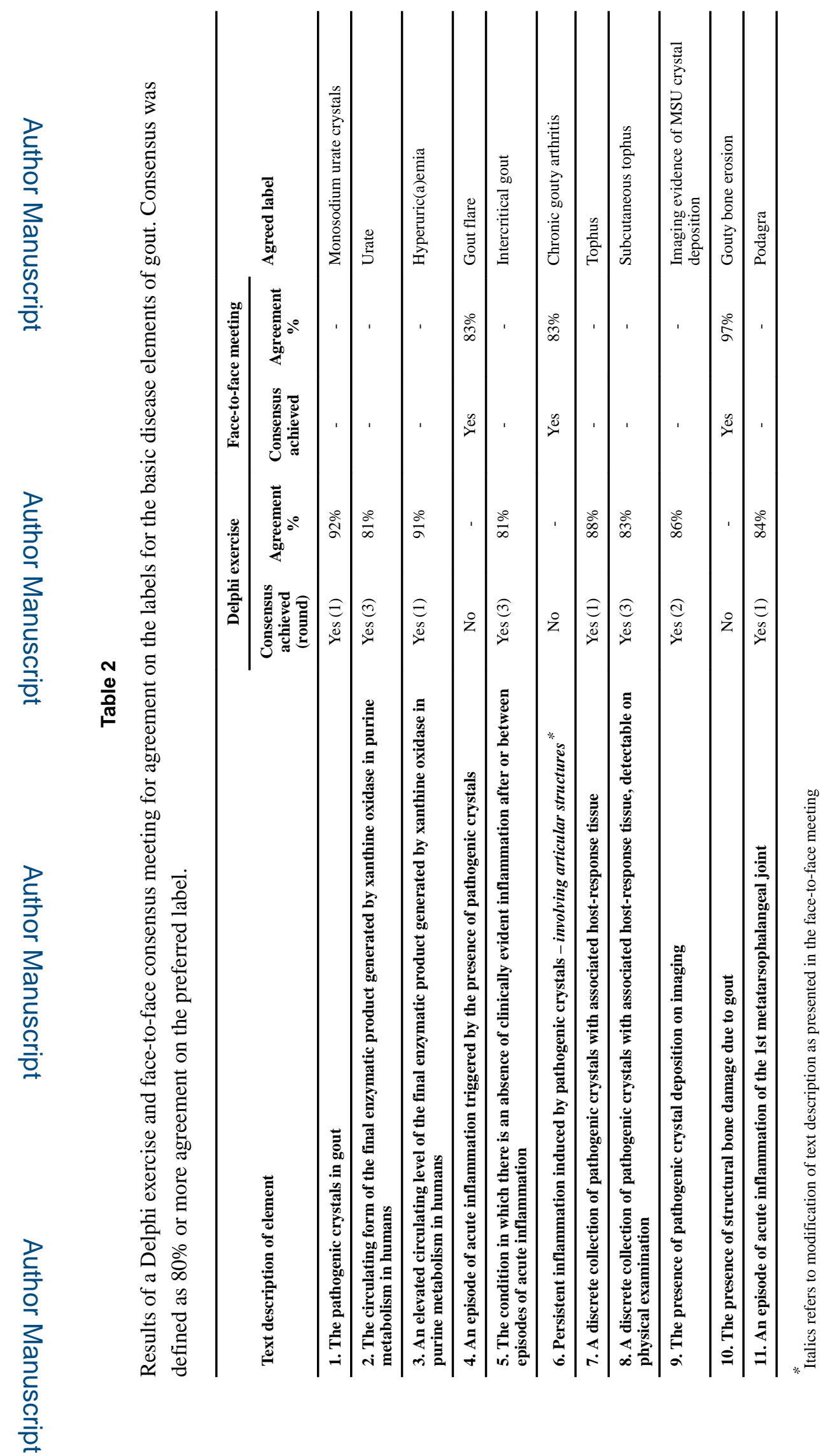

Arthritis Care Res (Hoboken). Author manuscript; available in PMC 2020 March 01. 


\section{Table 3}

G-CAN endorsed labels and definitions of the basic disease elements of gout following a Delphi exercise and face-to-face consensus meeting.

\begin{tabular}{ll}
\hline Consensus label & Consensus definition \\
\hline 1. Monosodium urate crystals & The pathogenic crystals in gout (chemical formula: $\mathrm{C}_{5} \mathrm{H}_{4} \mathrm{~N}_{4} \mathrm{NaO}_{3}$ ). \\
\hline 2. Urate & $\begin{array}{l}\text { The circulating form of the final enzymatic product generated by xanthine oxidase in purine } \\
\text { metabolism in humans (chemical formula: } \mathrm{C}_{5} \mathrm{H}_{3} \mathrm{~N}_{4} \mathrm{O}_{3}{ }^{-} \text {). }\end{array}$ \\
\hline 3. Hyperuric(a)emia & Elevated blood urate concentration over the saturation threshold. \\
\hline 4. Gout flare & A clinically evident episode of acute inflammation induced by monosodium urate crystals. \\
\hline 5. Intercritical gout & $\begin{array}{l}\text { The asymptomatic period after or between gout flares, despite the persistence of } \\
\text { monosodium urate crystals. }\end{array}$ \\
\hline 6. Chronic gouty arthritis & Persistent joint inflammation induced by monosodium urate crystals. \\
\hline 6a. G-CAN recommendation & The label 'chronic gout' should be avoided. \\
\hline 7. Tophus & An ordered structure of monosodium urate crystals and the associated host tissue response. \\
\hline 8. Subcutaneous tophus & A tophus that is detectable by physical examination. \\
\hline $\begin{array}{l}\text { 9. Imaging evidence of monosodium urate } \\
\text { crystal deposition }\end{array}$ & Findings that are highly suggestive of monosodium urate crystals on an imaging test. \\
\hline 10. Gouty bone erosion & $\begin{array}{l}\text { Evidence of a cortical break in bone suggestive of gout (overhanging edge with sclerotic } \\
\text { margin). }\end{array}$ \\
\hline 11. Podagra & A gout flare at the $1^{\text {st } \text { metatarsophalangeal joint. }}$ \\
\hline
\end{tabular}

